\title{
Artificial Intelligence in Pharmacy
}

\author{
Sudipta Das ${ }^{1, *}$, Rimi Dey ${ }^{1}$, Amit Kumar Nayak² \\ 1Department of Pharmaceutics, Netaji Subhas Chandra Bose Institute of Pharmacy, Nadia, West Bengal, INDIA. \\ ${ }^{2}$ Department of Pharmaceutics, Seemanta Institute of Pharmaceutical Sciences, Mayurbhanj, Odisha, INDIA.
}

\begin{abstract}
Artificial Intelligence (Al) focuses in producing intelligent modelling, which helps in imagining knowledge, cracking problems and decision making. Recently, Al plays an important role in various fields of pharmacy like drug discovery, drug delivery formulation development, polypharmacology, hospital pharmacy, etc. In drug discovery and drug delivery formulation development, various Artificial Neural Networks (ANNs) like Deep Neural Networks (DNNs) or Recurrent Neural Networks (RNNs) are being employed. Several implementations of drug discovery have currently been analysed and supported the power of the technology in quantitative structure-property relationship (QSPR) or quantitative structure-activity relationship (QSAR). In addition, de novo design promotes the invention of significantly newer drug molecules with regard to desired/optimal qualities. In the current review article, the uses of Al in pharmacy, especially in drug discovery, drug delivery formulation development, polypharmacology and hospital pharmacy are discussed.
\end{abstract}

Key words: Artificial intelligence, Artificial neural network, Drug discovery, Drug delivery research, Hospital pharmacy.

\section{INTRODUCTION}

Artificial Intelligence (AI) is a stream of science related to intelligent machine learning, mainly intelligent computer programs, which provides results in the similar way to human attention process. ${ }^{1}$ This process generally comprises obtaining data, developing efficient systems for the uses of obtained data, illustrating definite or approximate conclusions and selfcorrections/adjustments. ${ }^{2}$ In general, AI is used for analyzing the machine learning to imitate the cognitive tasks of individuals., ${ }^{2,3}$ AI technology is exercised to perform more accurate analyses as well as to attain useful interpretation. ${ }^{3}$ In this perspective, various useful statistical models as well as computational intelligence are combined in the AI technology. ${ }^{4}$ The progress and innovation of AI applications are often associated to the fear of unemployment threat. However, almost all advancements in the applications of AI technology are being celebrated on account of the confidence, which enormously contributes its efficacy to the industry.

Recently, AI technology becomes a very fundamental part of industry for the useful applications in many technical and research fields. ${ }^{3,4}$ The emergent initiative of accepting the applications of AI technology in pharmacy including drug discovery, drug delivery formulation development and other healthcare applications have already been shifted from hype to hope. ${ }^{5,6}$ The uses of AI models also make possible to predict the in vivo responses, pharmacokinetic parameters of the therapeutics, suitable dosing, etc. ${ }^{2,7}$ According to the importance of pharmacokinetic prediction of drugs, the uses of in silico models facilitate their effectiveness and inexpensiveness in the drug research. ${ }^{8}$ There are two key classes of AI technology developments. ${ }^{9}$ The first one comprises the conventional computing methodologies including expert systems, which are capable of simulating the human experiences and illustrating the conclusions
Submission Date: 12-05-2020; Revision Date: 03-09-2020; Accepted Date: 02-01-2021

DOI: 10.5530/ijper.55.2.68 Correspondence: Dr. Sudipta Das Associate Professor, Department of Pharmaceutics, Netaji Subhas Chandra Bose Institute of Pharmacy, Chakdaha, Nadia-741222, West Bengal, INDIA. Phone: +919434196529 Email id: sudiptapharmacy6@gmail. com

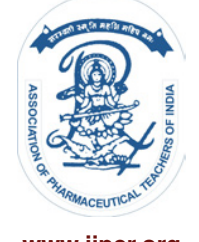

www.ijper.org 
from the principles, like expert systems. ${ }^{10}$ The second one comprises the systems, which can model the mode of brain functioning employing the artificial neural networks (ANNs). ${ }^{9}$ In specific, various ANNs like deep neural networks (DNNs) or recurrent neural networks (RNNs) control the evolutions of AI technology. In Merck Kaggle ${ }^{11}$ and NIH Tox21 challenge, ${ }^{12}$ DNN issues show the greater predictivity than the baseline machine learning methodologies., ${ }^{3,14}$ The machine learning employs suitable statistical methodologies with the capability to learn with or devoid of being unequivocally programmed..$^{13}$ In addition, de novo design promotes the invention of newer drug molecules with regard to optimal or desired qualities. ${ }^{14}$ In the current review article, the uses of AI in pharmacy, especially in drug discovery, drug delivery formulation development, polypharmacology and hospital pharmacy are discussed.

\section{Milestones in Al}

The first use of the phrase- 'Artificial Intelligence' was appeared in 1956. However, the concept of AI was employed since 1950 with the uses of problem-solving as well as symbolic methodologies. ${ }^{5}$ Important milestones in the area of the AI uses are presented in Table 1.

\section{Classification of $\mathrm{Al}$}

AI can be classified into two different ways: according to calibre and their presence

(Figure 1). ${ }^{15,16}$ According to their ability, AI can be categorized as:

i) Artificial Narrow Intelligence (ANI) or Weak AI: It performs a narrow range task, i.e., facial identification, steering a car, practicing chess, traffic signalling, etc.

\begin{tabular}{|c|c|}
\hline \multicolumn{2}{|c|}{ Table 1: Important milestones in the area of the Al } \\
uses.
\end{tabular}

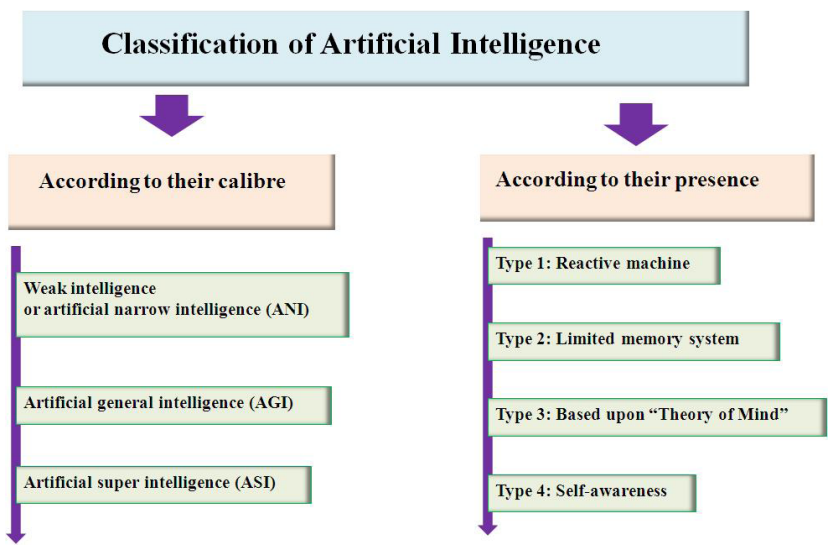

Figure 1: Classification of artificial intelligence.

ii) Artificial General Intelligence (AGI) or Strong AI: It performs all the things as humans and also known as human level AI. It can simplify human intellectual abilities and able to do unfamiliar task.

iii) Artificial Super Intelligence (ASI): It is smarter than humans and has much more activity than humans drawing, mathematics, space, etc.

According to their presence and not yet present, AI can be classified as follows:

i) Type 1: It is used for narrow purpose applications, which cannot use past experiences as it has no memory system. It is known as reactive machine. There are some examples of this memory, such as a IBM chess program, which can recognize the checkers on the chess playing board and capable of making predictions.

ii) Type 2: It has limited memory system, which can apply the previous experiences for solving different problems. In automatic vehicles, this system is capable of making decisions there are some recorded observations, which are used to record further actions, but these records are not stored permanently.

iii) Type 3: It is based upon "Theory of Mind". It means that the decisions that human beings make are impinged by their individual thinking, intentions and desires. This system is non-existing AI.

iv). Type 4: It has self-awareness, i.e., the sense of self and consciousness. This system is also non-existing AI.

\section{Neural networks and ANNs}

The learning algorithm of neural networks (from input data) takes two different forms mainly. The classes of neural networks are as follows (Figure 2): ${ }^{9,17}$

i) Unsupervised learning: Here the neural network is submitted with input data having recognised pattern. It is used for organizational purpose. 


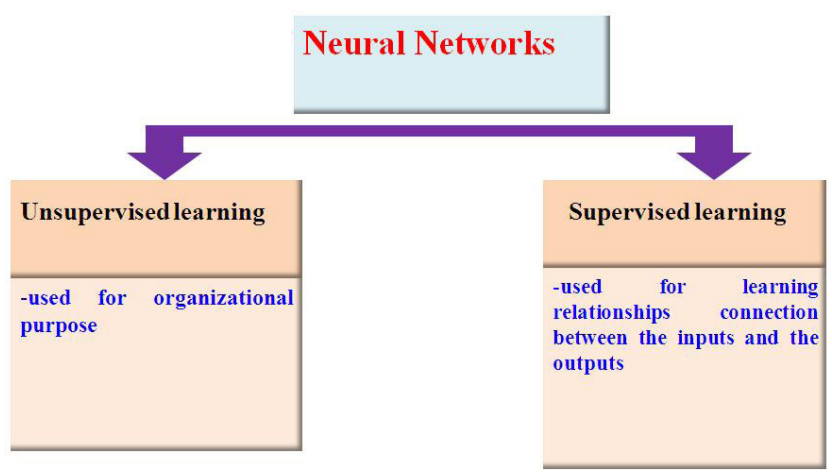

Figure 2: Classification of neural networks.

The unsupervised learning algorithm uses 'Self Organizing Map' or 'Kohonen'. 'This is known as very useful modeling for the searching of relationships amongst the complex data sets.

ii) Supervised learning: This kind of neural network is illustrated with the sequences of harmonizing inputs and outputs. It is used for learning relationshipconnection between the inputs and the outputs. It shows its usefulness in formulation to measure the cause and effects linking between input-output. It is the most frequently employed ANNs and is entirely linked with the back propagation learning rule. This learning algorithm is known as the outstanding methodology for the prediction as well as classification jobs. ${ }^{9}$

A simple mathematical processing unit called neuron is the main part of the neural network. ${ }^{17}$

Every input possesses an associated weight having relative importance and calculates the weighted sum of all the inputs as output. This output is then forwarded to another neuron after being modified by a transformation function. The whole processing is called a perceptron (a feed-forward system). A neural network having many neurons is organized into network architectures. ${ }^{9}$ The most famous and prosperous network is multilayer perceptron network. In this network system, the identical neurons are arranged in such a way that in one layer, the outputs are presented and in the subsequent layer, the inputs are presented. There are one or more secret layers, which can be introduced between the input and output layers. In theory, amount of secret layers can be attached according to solitary need. In practice, multiple layers are needed in case of applications with extensive nonlinear behaviour.

ANN is one of the computational modelling figured from hundreds of single units of artificial neurones associated with the constituents comprising the neural structure, which are known as processing elements as they participate in information processing. ${ }^{2,9,18} \mathrm{ANN}$ methodology presents a potential modeling procedure, in particular for the data sets of non-linear links commonly encountered in the pharmaceutical research. ${ }^{19-21}$ For the model specification analyses, ANNs don't necessitate acquaintance of the data source. However, they frequently have many weightages that should be analyzed. They also necessitate larger training sets. Additionally, ANNs can mix as well as add in both the literature and the investigational data to resolve the problems.

Recently, ANN models are being hybridized with other kinds of simpler models. ${ }^{22}$ For example, a recently proposed combination of neural networks and logistic regression allow the generation of hybrid linear/ nonlinear classification surfaces and the identification of possible strong interactions that may exist between the attributes (also known as covariates in the Logistic Regression literature) which define the classification problem. All these hybrid models perform reasonable well for a given set of databases. ${ }^{22}$

The prospective uses of ANNs in pharmacy are wideranged from the data analyses via the modeling of pharmaceutical quality control. ${ }^{21,23} \mathrm{ANNs}$ are also proved functional for the uses in the drug designing, especially in molecular modeling and QSAR. ${ }^{1,24}$ It is also used in formulation optimization processes for dosage form designing and in biopharmaceutical analyses, such as pharmacokinetic modeling, pharmacodynamic modeling, in vitro-in vivo correlation analysis, etc.,21-23,25

\section{Fuzzy logic and neurofuzzy logic}

According to the conventional logic, proposal may be true or false. The hypothesis behind the logic lies either in or totally outside the "true" set. When the hypothesis lies within the "true" set, the membership function is denoted as " 1 " and when the hypothesis lies outside the "true" set, the membership function is denoted as " 0 ". The basic concept of fuzzy logic is promoted by Lotfi Zadeh in the 1960 s. $^{26}$ In contrast to the conventional logic, the fuzzy logic is not limited to be 0 or 1 . However, any continuous value in-between these limits can be taken here. When $20^{\circ} \mathrm{C}$ temperature is taken as "comfortable", according to the conventional logic temperature of 19 or $21^{\circ} \mathrm{C}$, which remain outside this set, are "uncomfortable". But, according to fuzzy logic, $17^{\circ} \mathrm{C}$ may obtain a membership of 0.4 in the "hot" set as well as 0.6 in the "cold" set. This logic is very useful in process control. ${ }^{26}$ For the automated circulations by the arteries and venous, the automated system based on fuzzy logic for drug releasing has been framed and analyzed. ${ }^{27}$ Fuzzy hemodynamic management modules have already been employed for the assessment of the condition of patients to report the regulation of the arterial as well 
as pulmonary pressures. This can be used to monitor the cardiac output of patients. The fuzzy logic-based automated system offers a comparative faster reaction and more effectual haemodynamic control. ${ }^{27}$ In addition, the uses of supervisory-fuzzy rule-dependent adaptive control system is considered as a potential way for controlling the multiple drug hemodynamic process. ${ }^{28}$

When the fuzzy logic system is strongly combined with a neural network, it is called as neurofuzzy logic system. Here, the capability of neural networks of learning from data and the ability of fuzzy logic of expressing complex concepts intuitively are combined properly. It has data mining capability. The neurofuzzy logic also presents neural network having 2 extra layers for the fuzzification of inputs as well as defuzzification of outputs. In a research, the simulation of probucol absorption via the lipid formulations has been studied by means of neurofuzzy networkings. ${ }^{29}$ According to the outcome of the research, the probucol releasing rate from the lipid formulations was found to be significantly lesser in comparison with that of the self-emulsifying formulations. The adaptive neurofuzzy network model together with in vitro-in vivo correlation tool demonstrated the competent predictive presentation and the prospective for the development of complex relationships as well as interpolates the pharmacokinetic constraints. ${ }^{29}$

\section{Principal component analysis (PCA)}

PCA is another AI based model for decreasing the dataset-dimensionality by preserving as much 'variability' (i.e., statistical information) as possible and at the same time, PCA modelling minimizes the loss of information. PCA modelling translates into searching newer variables, which are linear functions of those in the original dataset by generating newer uncorrelated variables so that maximize the variance, successively. ${ }^{30}$ Searching of such newer variables, the principal components reduce the resolving of an eigenvector or eigenvalue problem. ${ }^{30,31}$ PCA can be based on either the covariance matrix or the correlation matrix and the main applications of PCA are descriptive in nature, rather than the inferential uses. Recent years, PCA is well-known for using as a 'hypothesis generating' AI tool generating a useful statistical mechanics frame for modelling of biological systems without the requirement for strong a priori theoretical assumptions, which makes PCA of paramount significance for drug discovery research by a systemic perspective overcoming too narrow reductionist approaches. ${ }^{32}$

\section{Support vector machine (SVM)}

SVM approach is based on the idea of a hyper plane classifier or linearly separability. It is mostly dependent on Statistical Learning Theory (SLT). The aim of SVM is to discover a linear optimal hyper plane in order to that the margin of separation in-between the two classifications is maximized..$^{33}$ SVM approach has recently been employed in many applications as it encompasses some potential benefits over various conventional machine learning methods. The important benefits of SVM approach are identified as: ${ }^{34}$

i) The solution of SVM approach is exclusive, optimal and global since the training of a SVM is done by solving a linearly confined quadratic problem.

ii) Only two free parameters are required to be chosen. These are known as: the kernel parameter and the upper bound parameter.

iii) SVM approach is capable of facilitating excellent generalization performance and good quality robustness.

Recent years, SVM approach has been employed for structure-activity relationship analysis and it proves its potential in drug discovery field. ${ }^{35,36}$ Burbidge et al. (2001) tested a benchmark test, where SVM approach was compared to several currently used machine learning techniques in the drug design field. ${ }^{37}$ The results of this research demonstrated that the SVM approach is significantly better as compared to these tested machine learning techniques currently used in drug designing, bar a manually capacity-controlled neural network, which takes considerably longer to train.

\section{Hammeistern Weiner (HW)}

HW modeling approach is one of the unrelated to physiological AI models. ${ }^{38}$ This is more flexible and better able to adapt to data, which results in a superior fitting when compared to different methods. ${ }^{38,39}$ HW model is implemented if a cascade of two static nonlinear blocks and one linear block are employed. Only the linear block contains the dynamic elements. ${ }^{39}$

In a research, Shokrollahi et al. (2018) evaluated the use of a nonlinear Hammerstein-Wiener modeling in the development and control of a magneto-rheological fluid haptic device, which can be potentially used for robotic bone biopsy. ${ }^{40}$ The validation testing performed in this research found that the HW modeling is able to predict the performance of the magneto-rheological fluid device with an accuracy of $95 \%$ and the HW modeling is capable of eliminating the hysteresis in a closed-loop control system.

\section{Advantages of Al technology}

The potential advantages of AI technology are as follows: ${ }^{6,15,41}$ 
i) Error minimization: $\mathrm{AI}$ assists to decrease the errors and increase the accuracy with more precision. Intelligent robots are made of resistant metal bodies and capable of tolerating the aggressive atmospheric space, therefore, they are sent to explore space.

ii) Difficult exploration: AI exhibits its usefulness in the mining sector. It is also used in the fuel exploration sector. AI systems are capable of investigating the ocean by defeating the errors caused by humans.

iii) Daily application: AI is very useful for our daily acts and deeds. For examples, GPS system is broadly used in long drives. Installation of AI in Androids helps to predict what an individual is going to type. It also helps in correction of spelling mistakes.

iv) Digital assistants: Now-a-days, the advanced organizations are using AI systems like 'avatar' (models of digital assistants) for the reduction of human needs. The 'avatar' can follow the right logical decisions as these are totally emotionless. Human emotions and moods disturb the efficiency of judgement and this problem can be overcome by the uses of machine intelligence.

v) Repetitive tasks: In general, human beings can perform single task at a time. In contrast to the human beings, machines are capable of performing multi-tasking jobs and can analyze more rapidly in comparison to the human beings. Various machine parameters, i.e., speed and time can be adjusted according to their requirements.

vi) Medical uses:In general, the physicians can assess the condition of patients and analyze the adverse effects and other health risks associated with the medication with the help of AI program. Trainee surgeons can gather knowledge by the applications of AI programs like various artificial surgery simulators (for examples, gastrointestinal simulation, heart simulation, brain simulation, etc.

vii) No breaks: Unlike human beings who have the capacity of working for $8 \mathrm{~h} /$ day with breaks, the machines are programmed in such a way that these are capable of performing the work in a continuous manner for long hours devoid of any kinds of confusions and boredom.

viii) Increase technological growth rate: AI technology is widely used in most of the advanced technological innovations worldwide. It is capable of producing different computational modelling programs and aims for the invention of the newer molecules. AI technology is also being used in the development of drug delivery formulations. ix) No risk: In case of working at the risky zone like fire stations, there are huge chances of causing harm to the personnel engaged. For the machine learning programs, if some mishap happens then broken parts can be repairable.

x) Acts as aids: AI technology has played a different function by serving children as well as elders on a $24 \times 7$ basis. It can perform as teaching and learning sources for all.

xi) Limitless functions: Machines are not restricted to any boundaries. The emotionless machines can do everything more efficiently and, also produce more accurately than the human beings.

\section{Disadvantages of Al technology}

The important disadvantages of AI technology are as follows: $: 15,41$

i) Expensive: The launch of AI causes huge money consumption. Complex designing of machine, maintenance and repairing are highly cost effective. For the designing of one AI machine, a long period of time is required by the $\mathrm{R} \& \mathrm{D}$ division. AI machine needs updating the software programmes, regularly. The reinstallations as well as recovery of the machine consume longer time and huge money.

ii) No replicating bumans: Robots with the AI technology are associated with the power of thinking like human and being emotionless as these add some advantages to perform the given task more accurately without any judgement. If unfamiliar problems arise, robots cannot take the decision and provide false report.

iii) No improvement with experience: Human resource can be improved with experiences. In contrast, machines with AI technology cannot be enhanced with experience. They are unable to identify which individual is hard working and which one is nonworking.

iv) No original creativity: Machines with AI technology have neither sensitivity nor the emotional intelligence. Humans have the ability to hear, see, feel and think. They can use their creativity as well as thoughts. These features are not achievable by the uses of machines.

v) Unemployment: The widespread uses of AI technology in all the sectors may cause large scale unemployment. As because of the undesirable unemployment, human workers may lose their working habits and creativity. 


\section{Al in hospital pharmacy}

There are several applications of AI in hospital pharmacybased health care system in organizing dosage forms for individualized patients, selection of the suitable or available administration routes or treatment policies. ${ }^{6,15}$

i) Maintaining of medical records: Maintenance of the medical records of patients is complicated task. The collection, storage normalizing, tracing of data are made easy by implementing the AI system. Google Deep Mind health project (developed by Google) assists to excavate the medical records in short span of time. Hence, this project is a useful one for better and faster health care. The Moor fields Eye hospital NHS is assisted by this project for improvement of eye treatment.

ii) Treatment plan designing: the designing of effective treatment plans is possible with the help of AI technology. ${ }^{15}$ When any critical condition of patient arises and selection of suitable treatment plan becomes difficult, then the AI system is necessary to control the situation. All the previous data and reports, clinical expertise, etc., are considered in the designing of treatment plan as suggested by this technology. A program is launched by IBM Watson to help oncologists.

iii) Assisting in repetitive tasks: AI technology also assists in some repetitive tasks, such as examining the X-ray imaging, radiology, ECHO, ECG, etc., for the detection and identification of the diseases or disorders. ${ }^{15}$ Medical Sieve (an algorithm launched by the IBM) is "cognitive assistant" having good analytical and reasoning ability. A medical start-up is necessary for the improvement of patient condition by combining deep learning with medical data. A specialized computer program is available for each body part and used in specific of disease conditions. Deep learning can be employed for almost all types imaging analyses, such as X-ray, CT scan, ECHO, ECG, etc.

iv) Health support and medication assistance: Recent years, the uses of AI technology are recognized efficient in health support services and also, for the medication assistances. ${ }^{6}$ Molly (a start-up designed virtual nurse) receives a pleasant voice along with a cordial face. Its aim of it is for helping patients to guide the treatment of patients as well as supporting them with chronic condition between the doctor's visits. Ai Cure, is an app existing in Smartphone's webcam, monitors patients and assists them to control their conditions.

v) AI helps to people in health care system: It is capable of collecting and comparing the data from social awareness algorithms. ${ }^{6}$ The vast information recorded in healthcare system involves the medical history of the patients along with the treatment history profile from the birth, habits as well as life-styles of the patients.

\section{Al approaches for drug discovery}

The drug discovery procedure begins from the available results attained from different resources like highthroughput screening modelling, fragment screening modelling, computational modelling and existing data reported. ${ }^{1,24}$ A schematic outline of the drug discovery procedure is shown in Figure 3. In drug discovery procedure, the structural characterization of drug molecules can directly or indirectly be analyzed by computer-assisted design approaches and after this, organic synthesis of drug molecules is done. The synthesized drug molecules or collected drug compounds are subjected to high throughput screening in primary assay and then, these are counter screened and evaluated for their bioavailability in secondary assays along with successful structure activity relationship (SAR) analysis. The drug discovery interchanges amongst induction and deduction processes. Thus, the interchangeable cycle of inductive-deductive process ultimately guides to attain the optimized lead molecules. ${ }^{1}$ The automation of specific portions of the inductive-deductive cycle decreases the unpredictability and inaccuracy; thus, improves the effectiveness of drug discovery procedure. Chemical and pharmaceutical manufacturers examine and derive numerous patents as well as genomic data-based scientific science information by applying the deep learning software, e.g., "NVIDIA DGX-1". Human beings cannot operate total available information for the advancement of scientific research. AI supercomputers are able to receive and examine the information for identification

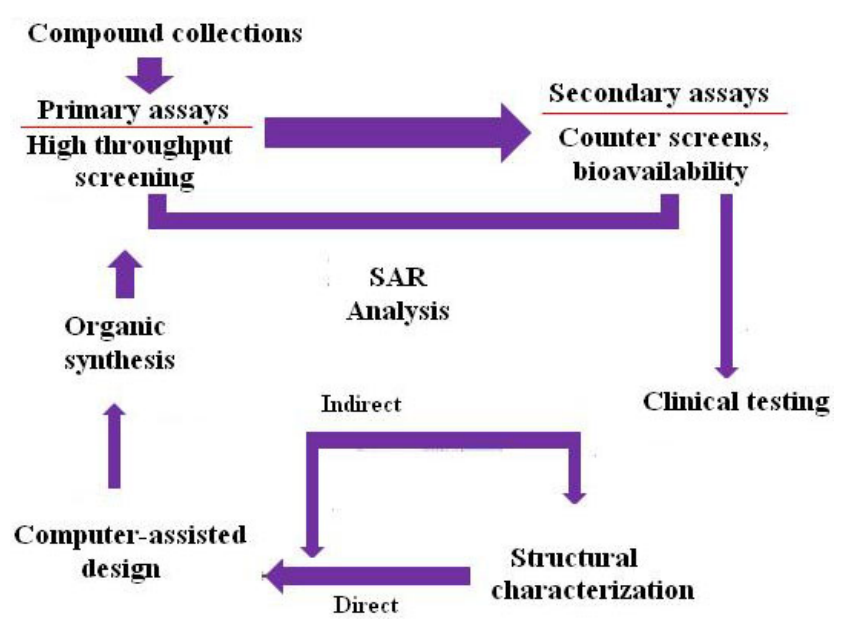

Figure 3: A schematic outline of the drug discovery procedure. 
the association in-between the compounds to offer newer drug molecules. ${ }^{9,42}$

The applications of AI in drug discovery process concerns the use of chemical space. ${ }^{1,42}$ In fact, the chemical space offers the phase for the identification of new molecules since it is achievable to computationally itemise the desired molecules. ${ }^{43}$ In addition, the machine learning and related predictive tools also help for the identifications of target-specific effective molecules. ${ }^{44}$ The process of selecting a successful new drug molecule from large quantity of pharmacological active chemical entities is the toughest part of the whole phenomenon. ${ }^{45}$ Benevolent AI is utilized for the purpose of processing fewer molecules with much more surety about their activity. In this regard, de novo design necessitate the understanding of organic chemistry for the synthesis of in silico molecules and the virtual screening modelling that perform as the replacements for many biochemical as well as biological testing to measure the efficiency as well as toxicity profiles. ${ }^{46-49}$ The aim of de novo design in the drug discovery is the invention of newer active molecules without the uses of reference molecules. ${ }^{47}$ Finally, the active learning algorithms permit the discovery of new molecules with the potential actions against the target-setting of diseases or disorders. Several in silico methods for the selection of profiles like ligand-based design approaches or molecular structure-based design approaches may be employed along with the accessible information on the small molecule modulator probes or the features of structural biology. ${ }^{50}$ In silico molecules is obtaining the next generation AI. There are various proposals and software solutions accessible for it. This design is not useful in drug discovery; but, it is connected to the generation of components possessing difficulty in synthesis. ${ }^{1}$

Although recursive neural networks are applied for de novo design, it is mainly introduced in the field of natural language processing. ${ }^{51}$ The sequential information is taken as input by recursive neural networks. As the molecular structures are encoded as a series of letters by SMILES strings, recursive neural networks are employed for generating the chemical structures of molecules. The neural networks are taught the grammar of SMILES strings by mean of training recursive neural networks with a wider setting of chemical compounds from the existing/available chemical compounds from existing compound collections (e.g., ChEMBL). The recursive neural networks have the capability for the processing of a high fraction of authentic SMILES strings. ${ }^{49}$ This approach is also applied for the creation of newer peptide structures. ${ }^{11}$ The reinforcement learning is also used in favour of the generated chemical molecule towards the desired characteristics. ${ }^{52}$ Another useful strategy is transfer learning used for generation of newer chemical structures processing a proper biological characteristics. This strategy possesses two steps. The step1 consists of training of the network for learning SMILES grammar. The step 2 consists of continued training with compounds possessing proper desired property. Such few extra epochs of training are enough in favour of the generation of newer compounds as into a chemical space possessed by the active molecules. Depending upon these approaches, in a work, synthesis of five molecules is done with the conformation of the design activity for four molecules against the nuclear hormone receptors. ${ }^{47}$

There is a fascinating method in the sphere of AI named 'Variational Autoencoder' having 2 neural networks: (i) encoder networks and (ii) decoder networks. The translation of chemical structures is illustrated by SMILES. This represents into a real-value continuous vector as the latent space and translation of vectors from which the latent space into the chemical structures are performed by encoder networks as well as decoder networks, respectively. The latent extent description is required by the authors for the training of a model which is dependent on synthetic accessibility score (SAS) and QED drug-likeness score. ${ }^{53}$ It is then possible to acquire a passage of molecules with upgraded target properties. In a published report, the comparison of 'Variational Autoencoder' with an 'Adversarial Autoencoder' was shown according to their performance. ${ }^{48}$ The 'Adversarial Autoencoder' has greater capability for producing appreciably more authentic molecular structures in comparison with that by the Variational Autoencoder' during their generation mode.

Generative adversarial networks (GANs) are capable for the designing of drug molecules. The technology can prepare photo-realistic pictures from the text representation. In a work, Kadurin et al. (2017) employed GAN for suggesting compounds having anticancer characteristics. ${ }^{54}$ Imagination or creations of new data can also be done by this technology based on the real data. Next generation AI is not dependent on the learning from large data sets. The new AI technology is capable to handle all the problems that are difficult to solve previously. ${ }^{41}$ The scientists and researchers are assisted by this new technology in the identification and selection of promising chemical agents on the basis of their efficacy, safety and selection of patients to the clinical trials. ${ }^{1,24}$ Therefore, AI is helpful in drug delivery because of its capability of prioritising the molecules according to the simplicity of syntheses or development of useful tools, which have been proved effectual for the most favourable synthetic technique. ${ }^{42}$ A list of important 
Table 2: List of important Al-based computer-assisted tools used in drug discovery.

\begin{tabular}{|c|c|c|}
\hline $\begin{array}{l}\text { Al-based computer- } \\
\text { assisted tools used in } \\
\text { drug discovery }\end{array}$ & Websites & Descriptions \\
\hline Chemputer & https://zenodo.org/record/1481731 & $\begin{array}{c}\text { More standardized set-up for reporting chemical } \\
\text { synthesis }\end{array}$ \\
\hline ODDT & https://github.com/oddt/oddt & $\begin{array}{l}\text { For use in chemo informatics and molecular } \\
\text { modelling }\end{array}$ \\
\hline ORGANIC & https://github.com/aspuru-guzik-group/ORGANIC & $\begin{array}{c}\text { Molecular generation tool to create molecules with } \\
\text { desired characteristics }\end{array}$ \\
\hline DeepChem & https://github.com/deepchem/deepchem & $\begin{array}{l}\text { A python-based Al tool for drug discovery } \\
\text { predictions }\end{array}$ \\
\hline DeepNeuralNet-QSAR & https://github.com/Merck/DeepNeuralNet-QSAR & Predictions of molecular activity \\
\hline $\begin{array}{l}\text { Neural Graph } \\
\text { Fingerprints }\end{array}$ & https://github.com/HIPS/neural-fingerprint & Property prediction of novel molecules \\
\hline Hit Dexter & http://hitdexter2.zbh.uni-hamburg.de & $\begin{array}{c}\text { Machine learning models for the prediction of } \\
\text { molecules, which might respond to biochemical } \\
\text { assays }\end{array}$ \\
\hline NNScore & http://rocce-vm0.ucsd.edu/data/sw/hosted/nnscore/ & $\begin{array}{l}\text { Analysis of neural network-based scoring function } \\
\text { for protein-ligand interactions }\end{array}$ \\
\hline DeepTox & www.bioinf.jku.at/research/DeepTox & Prediction of toxicity and biocompatibility \\
\hline PotentialNet & $\begin{array}{l}\text { https://pubs.acs.org/doi/full/10.1021/ } \\
\text { acscentsci.8b00507 }\end{array}$ & $\begin{array}{l}\text { Ligand-binding affinity prediction based on a } \\
\text { graph convolutional neural network }\end{array}$ \\
\hline REINVENT & https://github.com/MarcusOlivecrona/REINVENT & $\begin{array}{c}\text { Molecular de novo design using RNN and } \\
\text { reinforcement learning }\end{array}$ \\
\hline DeltaVina & https://github.com/chengwang88/deltavina & $\begin{array}{l}\text { A scoring function for rescoring protein-ligand } \\
\text { binding affinity }\end{array}$ \\
\hline AlphaFold & https://deepmind.com/blog/alphafold & Prediction of protein 3D structure prediction \\
\hline
\end{tabular}

AI-based computer-assisted tools used in drug discovery is presented in Table 2 .

\section{Al approaches for development of drug delivery systems}

Generally, the designing of drug delivery systems is related to some disadvantages like prediction of the relationship amongst the formulation factors and responses. ${ }^{2,55}$ This is also related to the therapeutic outcomes and the unpredicted occurrences. In the designing of different kinds of intelligent drug releasing systems, the on-demand dose adjustment or the rates of drug releasing, targeted releasing and drug stability are the important factors. ${ }^{56}$ Concerning the self-monitoring systems for releasing of drugs, the suitable algorithms are useful for controlling the quantity as well as the period of drug releasing. ${ }^{2}$ Therefore, AI approaches are useful for the prediction of the drug dosing efficacy and drug delivery potential of the drug delivery dosage forms.

Solid dispersions: The ANN modeling combined with experimental design has been employed to develop solid dispersions of carbamazepine using poloxamer 188 andSoluplus ${ }^{\circledR} .{ }^{57}$ The aim of the preparation of carbamazepine solid dispersions was to improve the carbamazepine solubility and dissolution rate. These carbamazepine-Soluplus ${ }^{\circledR}$-poloxamer 188 solid dispersions were synthesized via the solvent casting technique. In a research, a modeling of ANN (a feed-forward back propagation) with the logistic sigmoid activation function has already been employed for the analyses of linking amongst different variables as well as dissolution properties for the optimization of dissolution rate of drug. ${ }^{58}$ In this work, to prepare the solid dispersions of drug, poly (vinyl pyrrolidone)/polyethylene glycol mixtures were used as carriers. The applied ANNsassisted modeling established an appropriate prediction for the solid dispersion preparations of drugs with desired dissolution properties with along-standing physical stability. ${ }^{58}$

Emulsions and microemulsions: ANNs have also been utilized for the formulation development of stable emulsions (oil/water). ${ }^{59}$ The optimization of the fatty alcohol concentration to formulate emulsions (oil/water) was analyzed in this work. The independable variables (factors) analyzed in this work were concentrations of lauryl alcohol and time. The dependable variables (responses) were droplet size, zeta potential, viscosity 
and conductance. On the basis of validation testing, ANN-predicted values were found in excellent correlation with the data obtained from the experiment. ${ }^{59}$ ANNs have also been applied in the formulation designing of microemulsions, where the prediction of precision based on the microemulsion nature from the formula was easily analyzed. ${ }^{60}$ By means of the mixture of genetic algorithms and evolutionary ANNs, interior structural features and the microemulsion nature have also been forecasted with the high level of precision. In another work, an ANN modeling has been employed to forecast the formulation of stable microemulsions loaded with antitubercular drugs like rifampicin and isoniazid for oral administrations. ${ }^{61}$ Data obtained from the constructed pseudo-ternary phase triangle-diagrams presenting the oil components and the surfactant mixture were used for the testing as well as validation of the ANN modeling.

Tablets: In the designing of matrix tablets, static and dynamic ANNs have been applied for the dissolution profile modeling of different matrix tablets. ${ }^{62} \mathrm{In}$ this work, Monte Carlo simulations and the genetic algorithms optimizer tool were applied for these modelings based on ANN algorithm. The researcher used the Elman dynamic neural networks and decision trees, which appropriately predicted the dissolution properties of hydrophilic as well as lipid-based matrix tablets exhibiting controlled drug releasing pattern. As compared to the majority of commonly employed multilayer perceptron and static networks, the Elman neural networks-based modeling demonstrated the efficient modeling of drug releasing patterns by various formula of hydrophilic as well as lipid-based matrix tablets. ${ }^{6}$ In a research, matrix tablets for sustained release of an antidiabetic drug, metformin $\mathrm{HCl}$, was developed by means of multilayer perceptron with feed forward back propagation technique. ${ }^{63}$ The in vitro metformin $\mathrm{HCl}$ releasing pattern by the matrix tablets was optimized to develop the optimized formulations. The independent variables (factors) and dependable variables (responses) were analyzed for network training. In addition, the leave-oneout technique was employed for the model validation process by means of several trials. In another work, ANNs was applied for the formulation optimization of nimodipine matrix tablets for controlled releasing application. ${ }^{64} \mathrm{~A}$ combination of ANN-based modeling and statistical optimization process has been employed for the formulation designing of glipizide releasing osmotic pump tablets. ${ }^{65}$ In addition to the dissolution testing of these glipizide releasing osmotic pump tablets, the different formulation variables and process variables were optimized and analyzed by means of ANNs. A mixture of response surface methodology (RSM) and ANN-based modeling has been applied for the formulation optimization of osmotic tablets containing isradipine. ${ }^{66}$ The disparity amongst the predicted dissolution results and observed dissolution results for the optimized is radipine osmotic tablets was found to be within the experimentally caused error limits. In addition, the difference as well as similarity factors did not have any difference among the predicted dissolution results and observed dissolution results, demonstrating the appropriateness of ANN-based modeling to achieve the desired dissolution pattern for the formulation development of the controlled is radipine releasing osmotic tablets.

Multiparticulates (beads, microparticles and nanoparticles): By employing CAD/Chem softwareassisted modeling, multiparticulate beads of verapamil was developed.$^{67}$ In this work, the influences of various formulation variables as well as process variables on the in vitro verapamil releasing by the beads were analyzed. The in vitro verapamil releasing data for the optimized beads were found to be in the line of good agreement in comparison with that of the predicted results obtained by the ANN modeling. ${ }^{67}$ In a work, ANN modeling was applied to assess the influence of process variables on the papain (enzyme) entrapment within alginate-based beads for the improvement of stability as well as sitespecific release. ${ }^{68}$

The combination of ANN and RSM was applied to optimize alginate-based floating microspheres of aspirin, where the quantities of excipient materials, drug releasing and buoyant rate of microspheres were analyzed. ANN model was more precisely predicted in vitro aspirin releasing pattern in comparison with that of RSM. ${ }^{69}$ In a work, both the ANN model and factorial model as multivariate methods were used to develop the polymeric microspheres of verapamil $\mathrm{HCl}^{70}$ The mutual impacts of external phase $\mathrm{pH}$, initial loading of verapamil $\mathrm{HCl}$ and concentration of polymer used on various properties of microspheres was analyzed. The results of the study clearly demonstrated that ANN model showed the better fitting abilities with comparatively less biased and more accurate predictability in comparison to the factorial model.

RSM by full $3^{2}$ factorial design was employed for the formulation development of tamarind seed polysaccharide-alginate composite beads loaded with diclofenac sodium, where the impact of sodium alginate to tamarind seed polysaccharide ratio and cross-linker (calcium chloride) concentration as independent formulation 
variables on the drug encapsulation efficiency and drug release were statistically analyzed. ${ }^{71}$ Suitable polynomial equation involving independent formulation variables (factors) and their interactions was analyzed based on the estimation of several statistical parameters, such as correlation coefficient $\left(R^{2}\right)$, predicted correlation coefficient (predicted $R^{2}$ ), adjusted correlation coefficient (adjusted $R^{2}$ ) and predicted residual sum of squares (PRESS), provided by the Design-Expert ${ }^{\circledR}$ Software. In this research, two quadratic models were selected as suitable statistical modeling for optimization for drug encapsulation efficiency and drug release as responses, as because both the responses analyzed had the negligible PRESS values. PRESS is well-known statistical parameter for the measure of model fit to the data points and the smaller PRESS statistic indicates the better model fit to the data points. The predicted values obtained from the full $3^{2}$ factorial designs were coincided well with the observed responses. ${ }^{71}$

In a research, central composite design and RSM were employed for the formulation optimization of alginatemethylcellulose mucoadhesive microcapsules loaded with gliclazide, where the impact of sodium alginate to methylcellulose ratio and cross-linker (calcium chloride) concentration as independent formulation variables on the drug encapsulation efficiency and drug release were statistically analyzed. ${ }^{72}$ The statistical modeling was evaluated by one-way ANOVA $(p<0.05)$ and the model analysis was evaluated lack of fit and $R^{2}$ analysis and PRESS value for measured responses. In this work, for the formulation optimization, the quadratic model was selected based on the statistically insignificant lack of fit and smallest values of PRESS for encapsulation efficiency and drug release.

Malakar and Nayak (2012) developed a multiple-unit floating system, which was mainly low density oil (liquid paraffin)-entrapped calcium alginate-magnesium stearate beads loaded with ibuprofen by employing $2^{3}$ factorial design, where the effects of three independent process variables like amount of sodium alginate, magnesium stearate and liquid paraffin on density, ibuprofen entrapment and ibuprofen release were statistically analyzed by one-way ANOVA $(p<0.05) .{ }^{73}$ The results of ANOVA indicated that all models were significant $(p$ $<0.05$ ) for all the responses (namely density, ibuprofen entrapment and ibuprofen release).The in vitro drug release data were evaluated kinetically using various mathematical models.

The $R^{2}$ and root mean squared error (RMSE) values of these mathematical models were computed using KinetDS 3.0 Rev. 2010 software for accuracy as well as prediction capability of these mathematical models. When the respective $R^{2}$ were compared, the Korsmeyer-Peppas model was found as best-fit kinetic model. However, it was also observed to be closest to zero order model, Weibull model and Baker-Lonsdale model. Finally, the best fitting of the Korsmeyer-Peppas model was verified by comparing RMSE values for each tested models, where the minimum RMSE values $(0.12-0.68)$ was found.

In a work of development of albumin-loaded chitosan nanoparticles, ANN modeling was employed for the analyses of the impacts of various independable variables (factors) on the dependable variables (responses) like albumin loading efficiency and cytotoxicity profile. ${ }^{74}$ A 3 layer feed forward back propagation-based ANN modeling was studied for the development of nanoparticles of tri-block poly(lactide)-poly(ethylene glycol)poly(lactide) copolymer. ${ }^{75}$ In this work, on the basis of correlation coefficient $\left(R^{2}\right)$ and mean squared error (MSE) values, the best analytical model for prediction was chosen for training, test as well as data validation analysis. Amongst all the investigated variables, the concentration of polymer in the copolymer-based nanoparticle formulation was revealed as the most impacted factor. On the basis of central composite design (spherical), the formulation development of polymer-lipid hybrid nanoparticles of verapamil $\mathrm{HCl}$ was carried out, where the impacts of various formulation factors were analyzed. ${ }^{76}$ The multi-objective optimization of polymer-lipid hybrid nanoparticles of verapamil $\mathrm{HCl}$ was carried out employing the validated ANNs and continuous genetic algorithms and the analyses results indicated the better analytical capability of ANN model.

Some recent researches on the uses of AI technology in the formulation development of various kinds of drug delivery systems are also presented in Table 3.

\section{Al approaches in polypharmacology}

Now a day, 'one-disease-multiple-targets' concept governs over the 'one-disease-one -targets' concept for the advanced realization of pathological process in various disorders at their molecular basis. The phenomenon of 'one-disease-multiple-targets' is known as polypharmacology. ${ }^{110}$ There are numerous and useful databases, for examples, PubChem, KEGG, ChEMBL, ZINC, STITCH, Ligand Expo, PDB, Drug bank, Supertarget, Binding DB, etc, which are accessible for the accomplishment of a variety of important and useful information related to the structure of crystals, chemical features, biological properties, molecular pathways, binding affinities, disease concern, drug targets, etc. AI also helps to discover the databases to sketch polypharmacological molecules/agents. 


\section{Table 3: Some recent researches on the uses of Al in the development of drug delivery systems.}

\section{Drug delivery systems}

Ibuprofen-sustained release from tablets based on different cellulose derivatives

Novel granulated pellet-containing tablets and traditional pelletcontaining tablets

Ultrasonic release of drug from liposomes

Doxycycline hydroxypropyl- $\beta$-cyclodextrin inclusion complex

$$
\text { Oral disintegrating tablets }
$$

Agar nanospheres of Bupropion

Ophthalmic flexible nano-liposomes of pilocarpine $\mathrm{HCl}$

Floating tablets of rosiglitazone maleate

Nifedipine osmotic release tablets

Gelatin nanoparticles of diclofenac sodium

Hydroxyapatite (HAp)-ciprofloxacin bone-implants

Alginate-PVP K 30 microbeads of diclofenac sodium

Timolol-loaded ultradeformable nanoliposome formulations

Transferosomal gel for transdermal insulin delivery Besifloxacin $\mathrm{HCl}$ loaded liposomal gel

Transferosomal gel for transdermal delivery of risperidone Multiple-unit pellet system of prednisone

$\mathrm{pH}$-dependent mesalamine matrix tablets

Voriconazole loaded nanostructured lipid carriers based topical delivery system

Calcium alginate-gum Arabic beads of glibenclamide

Modified starch (cationized)-alginate beads of aceclofenac

Oil-entrapped sterculia gum-alginate beads ofaceclofenac

$$
\text { HAp-ofloxacin bone implants }
$$

Pioglitazone-loaded jackfruit seed starch-alginate beads

lonotropically-gelled mucoadhesive beads for oral metformin $\mathrm{HCl}$ delivery

Oral disintegrating tablet formulations

Sustained release matrix formulations of salbutamol sulfate

Jackfruit seed starch-alginate mucoadhesive beads of metformin $\mathrm{HCl}$

Jackfruit seed starch-pectin mucoadhesive beads of metformin $\mathrm{HCl}$

Emulsion-gelled floating beads of diclofenac sodium

Aceclofenac-loaded pectinate-poly (vinyl pyrrolidone) beads

Floating capsules containing alginate-based beads of salbutamol sulfate

Granulated pellet-containing tablets and traditional pellet-containing tablets
Al approaches used

Adaptive neural-fuzzy inference system

ANNs

ANNs

RSM, ANN and support vector machine (SVM) modeling

$$
\text { ANN and DNN }
$$

Genetic algorithm, ANN and RSM

$\mathrm{RSM}$ and ANN

ANNs

Mechanistic gastrointestinal simulation and ANN

Central composite design and ANNs

Quality by Design (QbD) and $2^{3}$ factorial design

Central composite design and RSM

ANN and multiple linear regression (MLR) analysis

$2^{3}$ factorial design and RSM

$3^{2}$ full factorial design and RSM

Central composite design and RSM

Box-Behnken design, RSM and ANN

ANN, multi-layer perception (MLP) algorithm and RMSE

Box-Behnken design and QbD

Central composite design and RSM

Central composite design and RSM

$3^{2}$ factorial design and RSM

$3^{2}$ factorial design and RSM

$3^{2}$ factorial design and RSM

$3^{2}$ factorial design and RSM

ANN

$3^{2}$ factorial design and RSM

$3^{2}$ factorial design and RSM

$2^{3}$ factorial design and RSM

$3^{2}$ factorial design and RSM

$3^{2}$ factorial design and RSM
Nayak and Pal (2013) ${ }^{101}$

Han et al. (2018) ${ }^{102}$

Chaibva et al. (2010) ${ }^{103}$

References

Rebouh et al. (2019) ${ }^{77}$

Huang et al. (2015) 78

Moussa et al. (2017)

Wang et al. (2013) ${ }^{80}$

Han et al. (2018) $)^{81}$

Zaki et al. (2015) $)^{82}$

Zhao et al. (2018) ${ }^{83}$

Güler et al. (2017) ${ }^{84}$

Koletti et al. (2020) ${ }^{86}$

Nayak et al. (2011) ${ }^{87}$

Nayak et al. (2011)

León Blanco et al.

$(2018)^{89}$

Malakar et al. (2012) $)^{90}$

Bhattacharjee et al. $(2020)^{91}$

Das et al. (2017) $)^{92}$

Manda et al. (2019) ${ }^{93}$

Khan et al. $(2020)^{94}$

Waghule et al. (2019) ${ }^{95}$

Nayak et al. (2012) $)^{96}$

Malakar et al. (2013) 97

Guru et al. (2013) ${ }^{98}$

Nayak et al. (2013) ${ }^{99}$

Nayak and Pal (2013) 104

Nayak and Pal (2013) 105

Nayak et al. (2013) ${ }^{106}$

Nayak et al. (2013) ${ }^{107}$

Malakar et al. (2014) ${ }^{108}$

Huang et al. (2015) ${ }^{109}$
Ilić et al. (2014) 85

Nayak et al. (2013) ${ }^{100}$ 


\section{CONCLUSION}

During past few years, a considerable amount of increasing interest towards the uses of AI technology has been identified for analyzing as well as interpreting some important fields of pharmacy like drug discovery, dosage form designing, polypharmacology, hospital pharmacy, etc., as the AI technological approaches believe like human beings imagining knowledge, cracking problems and decision making. The uses of automated workflows and databases for the effective analyses employing AI approaches have been proved useful. As a result of the uses of AI approaches, the designing of the new hypotheses, strategies, prediction and analyses of various associated factors can easily be done with the facility of less time consumption and inexpensiveness.

\section{ACKNOWLEDGEMENT}

Authors would like to acknowledge Principal and authorities of Netaji Subhas Chandra Bose Institute of Pharmacy, Nadia, West Bengal, India and Seemanta Institute of Pharmaceutical Sciences, Jharpokharia, Odisha, India.

\section{CONFLICT OF INTEREST}

The authors declare no conflicts of interest.

\section{ABBREVIATIONS}

AI: Artificial intelligence; AGI: Artificial general intelligence; ANI: Artificial narrow intelligence; ANN: Artificial neural network; DNN: Deep neural network; GAN: Generative adversarial network; QSAR: Quantitative structure-activity relationship; QSPR: Quantitative structure-property relationship; RNN: recurrent neural network; SAS: Synthetic accessibility score.

\section{REFERENCES}

1. Mak KK, Pichika MR. Artificial intelligence in drug development: Present status and future prospects. Drug Discov Today. 2019;24(3):773-80.

2. Hassanzadeh $P$, Atyabi F, Dinarvand $R$. The significance of artificial intelligence in drug delivery system design. Adv Drug Deliv Rev. 2019;151:169-90.

3. Russel S, Dewey D, Tegmark M. Research priorities for robust and beneficial artificial intelligence. Al Mag. 2015;36(4):105-14.

4. Duch W, Setiono R, Zurada JM. Computational intelligence methods for rulebased data understanding. Proc IEEE. 2004;92(5):771-805.

5. Dasta JF. Application of artificial intelligence to pharmacy and medicine. Hosp Pharm. 1992;27(4):319-22.

6. Jiang $\mathrm{F}$, Jiang $\mathrm{Y}$, Zhi H. Artificial intelligence in healthcare: Past, present and future. Stroke Vasc Neurol. 2017;2(4):230-43.

7. Gobburu JV, Chen EP. Artificial neural networks as a novel approach to integrated pharmacokinetic-pharmacodynamic analysis. J Pharm Sci. 1996;85(5):505-10.
8. Sakiyama Y. The use of machine learning and nonlinear statistical tools for ADME prediction. Expert Opin Drug Metab Toxicol. 2009;5(2):149-69.

9. Agatonovic-Kustrin S, Beresford R. Basic concepts of artificial neural network (ANN) modeling and its application in pharmaceutical research. J Pharm Biomed Anal. 2000;22(5):717-27.

10. Zhang ZH, Wang Y, Wu WF, Zhao X, Sun XC, Wang HQ. Development of glipizide push-pull osmotic pump controlled release tablets by using expert system and artificial neural network. Yao Xue Xue Bao. 2012;47(12):1687-95.

11. Ma J, Sheridan RP, Liaw A, Dahl GE, Svetnik V. Deep neural nets as a method for quantitative structure-activity relationships. J Chem Inf Model. 2015;55(2):263-74.

12. Mayr A, Klambauer G, Unterthiner T, Hochreither S. Deep Tox: Toxicity prediction using Deep Learning. Front Environ Sci. 2016;3:80.

13. Bishop CM. Model-based machine learning. Philos Trans A Math Phys Eng Sci. 2013;371(1984):20120222.

14. Merk D, Friedrich L, Grisoni F, Schneider G. De novo design of bioactive small molecules by artificial intelligence. Mol Inform. 2018;37(1-2):1-4.

15. Manikiran SS, Prasanthi NL. Artificial Intelligence: Milestones and Role in Pharma and Healthcare Sector. Pharma Times. 2019;51(1):10-1.

16. Cherkasov A, Hilpert K, Jenssen H, Fjell CD, Waldbrook M, Mullaly SC, et al. Use of artificial intelligence in the design of small peptide antibiotics effective against a broad spectrum of highly antibiotic resistant superbugs. ACS Chem Biol. 2009;4(1):65-74.

17. Haykin S. Neural Networks: A Comprehensive Foundation, $1^{\text {st }}$ ed. PrenticeHall PTR. NJ, United State. 1998.

18. Zupan J, Gasteiger J. Neural nets for mass and vibrational spectra. J Mol Struct. 1993;292:141-59.

19. Achanta AS, Kowalski JG, Rhodes CT. Artificial neural networks: Implications for pharmaceutical sciences. Drug Dev Ind Pharm. 1995;21(1):119-55.

20. Sakiyama $Y$. The use of machine learning and nonlinear statistical tools for ADME prediction. Expert Opin Drug Metab Toxicol. 2009;5(2):149-69.

21. Sutariya V, Groshev A, Sadana P, Bhatia D, Pathak Y. Artificial neural network in drug delivery and pharmaceutical research. Open Bioinf J. 2013;7(1):49-62.

22. Gutiérrez PA, Hervás-Martínez C. Hybrid Artificial Neural Networks: Models, Algorithms and Data. Advances in Computational Intelligence, Lecture Notes in Computer Science, Springer, Berlin, Heidelberg. 2011;6692.

23. Taskinen J, Yliruusi J. Prediction of physicochemical properties based on neural network modeling. Adv Drug Deliv Rev. 2003;55(9):1163-83.

24. Fleming $\mathrm{N}$. How artificial intelligence is changing drug discovery. Nature. 2018;557(7706):S55-7.

25. Sun $Y$, Peng $Y$, Chen $Y$, Shukla AJ. Application of artificial neural networks in the design of controlled release drug delivery systems. Adv Drug Deliv Rev. 2003;55(9):1201-15.

26. Zadeh LA. Fuzzy sets. Inform Control. 1965;8:338-53.

27. Huang JW, Roy RJ. Multiple-drug hemodynamic control using fuzzy decision theory. IEEE Transact Biomed Eng. 1998;45(2):213-28.

28. Held CM, Roy RJ. Multiple drug hemodynamic control by means of a supervisory fuzzy rule-based adaptive control system: Validation on a model. IEEE Trans Biomed Eng. 1995;42(4):371-85.

29. Fatouros DG, Nielsen FS, Douroumis D, Hadjileontiadis LJ, Mullertz A. In vitro-in vivo correlations of self-emulsifying drug delivery systems combining the dynamic lipolysis model and neuro-fuzzy networks. Eur J Pharm Biopharm. 2008;699(3):887-98.

30. Jolliffe IT, Jorge C. Principal component analysis: A review and recent developments. Phil Trans R Soc A. 2016;374(2065):20150202.

31. Jolliffe IT Principal Component Analysis" Springer-Verlag, (New York). 2002.

32. Giuliani A. The application of principal component analysis to drug discovery and biomedical data. Drug Discov Today. 2017;22(7):1069-76.

33. Burges CJC. A tutorial on support vector machines for pattern recognition. Data Mining Knowledge Discov. 1998;2(2):121-67.

34. Heba FE, Darwish A, Hassanien AE, Abraham A. Principle components analysis and support vector machine based intrusion detection system, $10^{\text {th }}$ International Conference on Intelligent Systems Design and Applications, Cairo. 2010;363-7.

35. Heikamp K, Bajorath J. Support vector machines for drug discovery. Expert Opin Drug Discov. 2014;9(1):93-104. 
36. Maltarollo VG, Kronenberger T, Espinoza GZ, Oliveira PR, Honorio KM. Advances with support vector machines for novel drug discovery. Expert Opin Drug Discov. 2019;14(1):23-33.

37. Burbidge $\mathrm{R}$, Trotter M, Buxton $\mathrm{B}$, Holden S. Drug design by machine learning: Support vector machines for pharmaceutical data analysis. Comput Chem. 2001;26(1):5-14.

38. Ljung L. Black-box models from input-output measurements. In Proceedings of the $18^{\text {th }}$ IEEE Instrumentation and Measurement Technology Conference. Rediscovering Measurement in the Age of Informatics (Cat. $\mathrm{No.01 \textrm {CH }}$ 37188), Budapest, Hungary. 2001;138-146.21.

39. Patcharaprakiti N, Kirtikara K, Chenvidhya D, Monyakul V, Muenpinij B. Modeling of single phaseinverter of photovoltaic system using system identification. In Proceedings of the 2010 Second International Conference on Computer and Network Technology, Bangkok, Thailand. 2010;462-6.

40. Shokrollahi E, Goldenberg AA, Drake JM, Eastwood KW, Kang M. Application of a nonlinear Hammerstein-Wiener estimator in the development and control of a magnetorheological fluid haptic device for robotic bone biopsy. Actuators. 2018;7(4):83.

41. Silver D, Schrittwieser J, Simonyan K. Mastering the game of Go without human knowledge. Nature. 2017;550(7676):354-9.

42. Lakdawala AS, Okafo G, Baldoni J, Palovich M, Sikosek T, Sahni V. Adapting drug discovery to artificial intelligence. Drug Target Rev. 2018;50-2.

43. Reymond JL, Deursen RV, Blum LC, Ruddigkeit L. Chemical space as a source for new drugs. Med Chem Commun. 2010;1(1):30-8.

44. Ferrero E, Dunham I, Sanseau P. In silico prediction of novel therapeutic targets using gene-disease association data. J Transl Med. 2017;15(1):182.

45. Katsila T, Spyroulias GA, Patrinos GP, Matsoukas MT. Computational approaches in target identification and drug discovery. Comput Struct Biotechnol J. 2016;14:177-84.

46. Yuan Y, Pei J, Lai L. Lig Builder 2: A practical de novo drug design approach. J Chem Inf Model. 2011;51(5):1083-91.

47. Merk D, Friedrich L, Grisoni F, Schneider G. De novo Design of Bioactive Small Molecules by Artificial Intelligence Daniel. Mol Inform. 2018;37(1-2):201700153.

48. Blaschke T, Olivecrona M, Engkvist O, Bajorath J, Chen H. Application of Generative Autoencoder in de nov Molecular Design. Mol Inform. 2018;37(12):201700123.

49. Gupta A, Müller AT, Huisman BJH, Fuchs JA, Schneider P, Schneider G. Generative Recurrent Networks for de nov Drug Design. Mol Inform. 2018;37(1-2):201700111.

50. Ferrero E, Dunham I, Sanseau P. In silico prediction of novel therapeutic targets using gene-disease association data. J Transl Med. 2017;15(1):182.

51. Muller AT, Hiss JA, Schneider G. Recurrent Neural Network Model for Constructive Peptide Design. J Chemlnf Model. 2018;58(2):472-9.

52. Olivecrona M, Blaschke T, Engkvist $\mathrm{O}$, Chen $\mathrm{H}$. Molecular de-novo design through deep reinforcement learning. J Cheminform. 2017;9(1):48

53. Ertl P, Schuffenhauer A. Estimation of synthetic accessibility score of druglike molecules based on molecular complexity and fragment contributions. J Cheminform. 2009;1(1):8.

54. Kadurin A, Nikolenko S, Khrabrov K, Aliper A, Zhavoronkov A. druGAN: An advanced generative adversarial autoencoder model for de novo generation of new molecules with desired molecular properties in silico. Mol Pharm. 2017;14(9):3098-104.

55. Yildirim O, Gottwald M, Schüler P, Michel MC. Opportunities and challenges for drug development: public-private partnerships, adaptive designs and big data. Front Pharmacol. 2016;7:461.

56. Shah N, Patel N, Patel KR. A sequential review on intelligent drug delivery system. J Pharm Sci Biosci Res. 2013;3(5):158-62.

57. Medarevic DP, Kleinebudde P, Djuris J, Djuric Z, Ibric S. Combined application of mixture experimental design and artificial neural networks in the solid dispersion development. Drug Devind Pharm. 2016;42(3):389-402.

58. Barmpalexis P, Koutsidis I, Karavas E, Louk D. Development of PVP/PEG mixtures as appropriate carriers for the preparation of drug solid dispersions by melt mixing technique and optimization of dissolution using artificial neural networks. Eur J Pharm Biopharm. 2013;85(3):1219-31.

59. Kumar KJ, Panpalia GM, Priyadarshini S. Application of artificial neural networks in optimizing the fatty alcohol concentration in the formulation of an O/W emulsion. Acta Pharm. 2011;61(2):249-56.
60. Podlogar F, Šibanc R, Gašperlin M. Evolutionary artificial neural networks as tools for predicting the internal structure of microemulsions. J Pharm Pharmaceut Sci. 2008;11(1):67-76.

61. Agatonovic-Kustrin S, Glass BD, Wisch MH, Alany RG. Prediction of a stable microemulsion formulation for the oral delivery of a combination of antitubercular drugs using ANN methodology. Pharm Res. 2003;20(11):1760-5.

62. Petrovic J, Ibric S, Betz G, Duric Z. Optimization of matrix tablets controlled drug release using Elman dynamic neural networks and decision trees. Int $\mathrm{J}$ Pharm. 2012;428(1-2):57-67.

63. Mandal U, Gowda V, Ghosh A, Bose A, Bhaumik U, Chatterjee B. Optimization of metformin $\mathrm{HCl} 500 \mathrm{mg}$ sustained release matrix tablets using artificial neural network (ANN) based on multilayer perceptrons (MLP) model. Chem Pharm Bull. 2008;56(2):150-5.

64. Barmpalexis P, Kanaze FI, Kachrimanis K, Georgarakis E. Artificial neural networks in the optimization of a nimodipine controlled release tablet formulation. Eur J Pharm Biopharm. 2010;74(2):316-23.

65. Zhang ZH, Wang Y, Wu WF, Zhao X, Sun XC, Wang HQ. Development of glipizide push-pull osmotic pump controlled release tablets by using expert system and artificial neural network. Yao Xue Xue Bao. 2012;47(12):1687-95.

66. Patel A, Mehta T, Patel M, Patel K, Patel N. Design porosity osmotic tablet for delivering low and $\mathrm{pH}$-dependent soluble drug using an artificial neural network. Curr Drug Deliv. 2012;9(5):459-67.

67. Vaithiyalingam S, Khan MA. Optimization and characterization of controlled release multi-particulate beads formulated with customized cellulose acetate butyrate dispersion. Int J Pharm. 2002;234(1-2):179-93.

68. Sankalia MG, Mashru RC, Sankalia JM, Sutariya VB. Papain entrapment in alginate beads for stability improvement and site-specific delivery: physicochemical characterization and factorial optimization using neural network modeling. AAPS Pharm Sci Tech. 2005;6(2):E209-22.

69. Zhang AY, Fan TY. Optimization of calcium alginate floating microspheres loading aspirin by artificial neural networks and response surface methodology. Beijing Da Xue Xue Bao Yi Xue Ban. 2010;42(2):197-201.

70. Labouta HI, El-khordagui LK, Molokhia AM, Ghaly GM. Multivariate modelling of encapsulation and release of an ionizable drug from polymer microspheres. J Pharm Sci. 2009;98(12):4603-15.

71. NayakAK, Pal D. Development of $\mathrm{pH}$-sensitive tamarind seed polysaccharidealginate composite beads for controlled diclofenac sodium delivery using response surface methodology. Int J Biol Macromol. 2011;49(4):784-93.

72. Pal D, Nayak AK. Development, optimization and anti-diabetic activity of gliclazide-loaded alginate-methyl cellulose mucoadhesive microcapsules. AAPS Pharm Sci Tech. 2011;12(4):1431-41.

73. Malakar J, Nayak AK. Formulation and statistical optimization of multiple-unit ibuprofen-loaded buoyant system using $2^{3}$-factorial design. Chem Eng Res Des. 2012;90(11):1834-46.

74. Baharifar H, Amani A. Size, loading efficiency and cytotoxicity of albuminloaded chitosan nanoparticles: An artificial neural networks study. J Pharm Sci. 2017;106(1):411-7.

75. Asadi H, Rostamizadeh K, Salari D, Hamidi M. Preparation of biodegradable nanoparticles of tri-block PLA-PEG-PLA copolymer and determination of factors controlling the particle size using artificial neural network. J Microencapsul. 2011;28(5):406-16.

76. Li Y, Abbaspour MR, Grootendorst PV, Rauth AM, Wu XY. Optimization of controlled release nanoparticle formulation of verapamil hydrochloride using artificial neural networks with genetic algorithm and response surface methodology. Eur J Pharm Biopharm. 2015;94:170-9.

77. Rebouh S, Lefnaoui S, Bouhedda M, Yahoum MM, Hanini S. Neuro-fuzzy modeling of ibuprofen-sustained release from tablets based on different cellulose derivatives. Drug Deliv Transl Res. 2019;9(1):162-77.

78. Huang Y, Yao Q, Zhu C, Zhang X, Qin L, Wang Q. Comparison of novel granulated pellet-containing tablets and traditional pellet-containing tablets by artificial neural networks. Pharm Dev Technol. 2015;20(6):670-5.

79. Moussa HG, Husseini GA, Abel-Jabbar N, Ahmad SE. Use of model predictive control and artificial neural networks to optimize the ultrasonic release of a model drug from liposomes. IEEE Transact Nanobiosci. 2017;16(3):149-56.

80. Wang Z, He Z, Zhang L, Zhang H, Zhang M, Wen X. Optimization of a doxycycline hydroxypropyl- $\beta$-cyclodextrin inclusion complex based on computational modeling. Acta Pharm Sin B. 2013;3(2):130-9. 
81. Han R, Yang Y, LiX, Ouyang D. Predicting oral disintegrating tablet formulations by neural network techniques. Asian J Pharm Sci. 2018;13(4):336-42.

82. Zaki MR, Varshosaz J, Fathi M. Preparation of agar nanospheres: Comparison of response surface and artificial neural network modeling by a genetic algorithm approach. Carbohydr Polym. 2015;122:314-20.

83. Zhao F, Lu J, Jin X, Wang Z, Sun Y, Gao D, et al. Comparison of response surface methodology and artificial neural network to optimize novel ophthalmic flexible nano-liposomes: Characterization, evaluation, in vivo pharmacokinetics and molecular dynamics simulation. Colloids Surf B Biointerf. 2018;172:288-97.

84. Güler GK, Eroğlu H. Development and formulation of floating tablet formulation containing rosiglitazone maleate using artificial neural network. J Drug Deliv Sci Technol. 2017;29:385-97.

85. Ilić $\mathrm{M}$, Đuriš $\mathrm{J}$, Kovačević I, Ibrić $\mathrm{S}$, Parojčić J. In vitro-in silico-in vivo drug absorption model development based on mechanistic gastrointestinal simulation and artificial neural networks: Nifedipine osmotic release tablets case study. Eur J Pharm Sci. 2014;62:212-8.

86. Koletti AE, Tsarouchi E, Kapourani A, Kontogiannopoulos KN, Assimopoulou AN Barmpalexis P. Gelatinnanoparticles for NSAID systemic administration: Quality by design and artificial neural networks implementation. Int J Pharm. 2020;578:1-14.

87. Nayak AK, Laha B, Sen KK. Development of hydroxyapatite-ciprofloxacin bone-implants using >>Quality by Design $<<$. Acta Pharm. 2011;61(1):25-36.

88. Nayak AK, Khatua S, Hasnain MS, Sen KK. Development of alginate-PVP K 30 microbeads for controlled diclofenac sodium delivery using central composite design. DARU J Pharm Sci. 2011;19(5):356-66.

89. León BJM, González RPL, Arroyo GCM, Cózar-Bernal MJ, Calle SM, Canca $\mathrm{OD}$, et al. Artificial neural networks as alternative tool for minimizing error predictions in manufacturing ultradeformable nanoliposome formulations. Drug Devlnd Pharm. 2018;44(1):135-43.

90. Malakar J, Sen SO, Nayak AK, Sen KK. Preparation, optimization and evaluation of transferosomal gel for transdermal insulin delivery. Saudi Pharm J. 2012;20(4):355-63.

91. Bhattacharjee A, Das PJ, Dey S, Nayak AK, Roy PK, Chakrabarti S, et al. Development and optimization of besifloxacin hydrochloride loaded liposomal gel prepared by thin film hydration method using $3^{2}$ full factorial design. Colloids Surf, A: Physicochem Eng Asp. 2020;585:124071.

92. Das B, Sen SO, Maji, Nayak AK, Sen KK. Transferosomal gel for transdermal delivery of risperidone: Formulation optimization and ex vivo permeation. J Drug Deliv Sci Technol. 2017;38:59-71.

93. Manda A, Walker RB, Khamanga SMM. An artificial neural network approach to predict the effects of formulation and process variables on prednisone release from a multipartite system. Pharmaceutics. 2019;11(3):109.

94. Khan AM, Hanif M, Bukhari NI, Shamim R, Rasool F, Rasul S, et al. Artificial neural network (ANN) approach to predict an optimized pH-dependent mesalamine matrix tablet. Drug Des Devel Ther. 2020;14:2435-48.
95. Waghule T, Rapalli VK, Singhvi G, Manchanda P, Hans N, Dubey SK, et al. Voriconazole loaded nanostructured lipid carriers based topical delivery system: $\mathrm{QbD}$ based designing, characterization, in-vitro and ex-vivo evaluation. J Drug Deliv Sci Technol. 2019;52:303-15.

96. Nayak AK, Das B, Maji R. Calcium alginate/gum Arabic beads containing glibenclamide: Development and in vitro characterization. Int J Biol Macromol. 2012;51(5):1070-8.

97. Malakar J, Nayak AK, Das A. Modified starch (cationized)-alginate beads containing aceclofenac: Formulation optimization using central composite design. Stärke. 2013;65(7-8):603-12.

98. Guru PR, Nayak AK, Sahu RK. Oil-entrapped sterculia gum-alginate buoyant systems ofaceclofenac: Development and in vitro evaluation. Colloids Surf B: Biointerf. 2013;104:268-75.

99. Nayak AK, Hasnain MS, Malakar J. Development and optimization of hydroxyapatite-ofloxacin implants for possible bone-implantable delivery in osteomyelitis treatment. Curr Drug Deliv. 2013;10(2):241-50.

100. Nayak AK, Pal D, Hasnain MS. Development, optimization and in vitro-in vivo evaluation of pioglitazone-loaded jackfruit seed starch-alginate beads. Curr Drug Deliv. 2013;10(5):608-19.

101. Nayak AK, Pal D. Ionotropically-gelled mucoadhesive beads for oral metformin $\mathrm{HCl}$ delivery: Formulation, optimization and antidiabetic evaluation. J Sci Ind Res. 2013;72:15-22.

102. Han R, Yang Y, LiX, Ouyang D. Predicting oral disintegrating tablet formulations by neural network techniques. Asian J Pharm Sci. 2018;13(4):336-42.

103. Chaibva F, Burton M, Walker RB. Optimization of salbutamol sulfate dissolution from sustained release matrix formulations using an artificial neural network. Pharmaceutics. 2010;2(2):182-98.

104. Nayak AK, Pal D. Formulation optimization of jackfruit seed starch-alginate mucoadhesive beads of metformin HCl. Int J Biol Macromol. 2013;59:264-72.

105. Nayak AK, Pal D. Blends of jackfruit seed starch-pectin in the development of mucoadhesive beads containing metformin $\mathrm{HCl}$. Int $\mathrm{J}$ Biol Macromol. 2013;62:137-45.

106. Nayak AK, Pal D, Malakar J. Development, optimization and evaluation of emulsion-gelledfloating beads using natural polysaccharide-blendfor controlled drug release. Polym Eng Sci. 2013;53(2):338-50.

107. Nayak AK, Kalia S, Hasnain MS. Optimization of aceclofenac-loaded pectinate-poly (vinyl pyrrolidone) beads by response surface methodology. Int J Biol Macromol. 2013;62:194-202.

108. Malakar J, Dutta P, Purokayastha SD, Dey S, Nayak AK. Floating capsules containing alginate-based beads of salbutamol sulfate: In vitro-in vivo evaluations. Int J Biol Macromol. 2014;64:181-9.

109. Huang Y, Yao Q, Zhu C, Zhang X, Qin L, Wang Q, et al. Comparison of novel granulated pellet-containing tablets and traditional pellet-containing tablets by artificial neural networks. Pharm Dev Technol. 2015;20(6):670-5.

110. Prescott JH, Lipka S, Baldwin S, JrSheppard NF, Maloney JM, Coppeta J, et al. Chronic, programmed polypeptide delivery from an implanted, multireservoir microchip device. Nature Biotechnol. 2006;24(4):437-8. 


\section{PICTORIAL ABSTRACT}

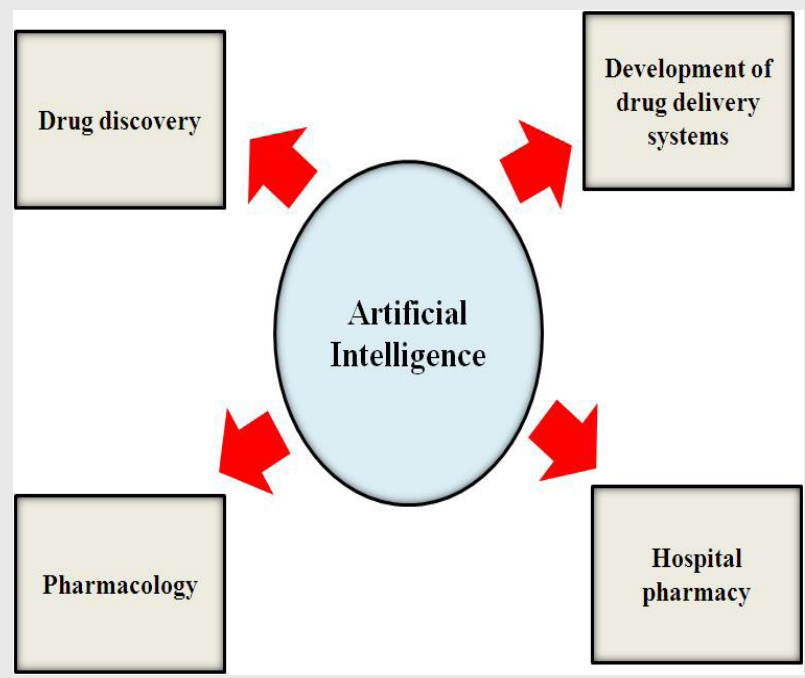

\section{SUMMARY}

Artificial Intelligence (Al) focuses in producing intelligent modelling, which helps in imagining knowledge, cracking problems and decision making. Recently, Al plays an important role in various fields of pharmacy like drug discovery, drug delivery formulation development, polypharmacology, hospital pharmacy, etc. Al technological approaches believe like human beings imagining knowledge, cracking problems and decision making. As a result of the uses of $\mathrm{Al}$ approaches, the designing of the new hypotheses, strategies, prediction and analyses of various associated factors can easily be done with the facility of less time consumption and inexpensiveness.

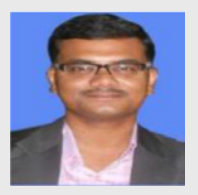

\section{About Authors}

Dr. Sudipta Das has obtained his Ph.D degree from Department of Pharmaceutical Technology, Jadavpur University, Kolkata, India. Presently he is working as Associate Professor at Netaji Subhas Chandra Bose Institute of Pharmacy. He has more than 100 research and review publications in both National and International reputed Journals. Author successfully guided one Ph.D student from Jadavpur University. He has already authored three books in the subject of Pharmaceutics. Many book chapters are also in his credit in Taylor and Francis group. The author is also life member of some professional bodies like Indian Pharmaceutical Association, Association of Pharmaceutical Teachers in India, Fellow of Indian Chemical Society and Associates of Institute of Chemist India. He conferred "Award for Teaching Excellence" in the year 2017 and also "Swami Vivekananda Excellence Award" on Science and Technology in the year 2019.

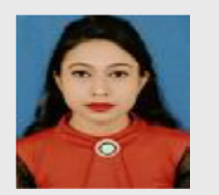

Ms. Rimi Dey has obtained B.Pharm degree from Netaji Subhas Chandra Bose Institute of Pharmacy, West Bengal, India. She has one research publication in International journal.

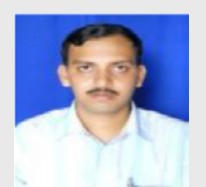

Dr. Amit Kumar Nayak is currently working as Associate Professor at Seemanta Institute of Pharmaceutical Sciences, Odisha, India. He has earned his Ph.D from IFTM University, Moradabad, U.P, India. He worked as Senior Research Associate at IIT, Kanpur in a CSIR sponsored project. Till date, he has authored over 120 research and review publications in various high impact peerreviewed journals and 102 book chapters and 9 books to his credit. He has received University Foundation Day Research Award-2019 by Biju Patnaik University of Technology, Odisha. Recently, he has been enlisted amongst top $2 \%$ scientists in a global list compiled by the Stanford University. Dr. Nayak is life member of Association of Pharmaceutical Teachers of India (APTI) and a Registered Pharmacist.

Cite this article: Das S, Dey R, Nayak AK. Artificial Intelligence in Pharmacy. Indian J of Pharmaceutical Education and Research. 2021;55(2):304-18. 